\title{
OPTIMIZING SUBJECT DESIGN, TIMING, AND FOCUS IN A DIVERSITY OF ENGINEERING COURSES THROUGH THE USE OF A LOW-COST ARDUINO SHIELD
}

\author{
Andres Gongora, Juan-Antonio Fernández-Madrigal, Ana Cruz-Martín, \\ Vicente Arévalo-Espejo, Cipriano Galindo-Andrades, Carlos Sánchez-Garrido, \\ Javier Monroy, Javier Fernández-Cañete
}

Machine Perception and Intelligent Robotics (MAPIR) research group, and Biomedical Research Institute of Malaga (IBIMA). University of Malaga (SPAIN)

\begin{abstract}
This paper describes the design, implementation and evaluation of a novel circuitry that extends the popular Arduino UNO microcontroller board to facilitate multiple educational activities in engineering courses. In particular, the aim of this board, the UMA-AEB, is to minimize the overhead that is usually imposed on the students before they can conduct the actual exercises, yet retain the valuable experiences that could otherwise not be acquired with simulated experiments or inflexible electronic training-benches. The UMA-AEB is also meant to bring analog and digital electronics closer to other common disciplines in engineering, such as high-level programming languages (e.g. MATLAB) or RealTime Operating Systems (e.g. FreeRTOS), by easing its connection to a personal computer for control and debugging. The features on the UMA-AEB range from simple input-output devices like a potentiometer, several buttons, eight individually addressable LEDs and a seven-segment display, to more advanced components like an SPI memory module for data-logging, a configurable resistiveinductive-capacitive (RLC) first and second order resonator, and a bidirectional analog-digital interface supporting -10 to $+10 \mathrm{~V}$, a common range for most college-level laboratory classes. The resulting design can be manufactured at a significantly lower cost than other similar devices (around $50 €$ not considering labor costs such as component-soldering and basic diagnoses), and both simulation and real tests have demonstrated that the obtained functionality agrees accurately enough with our design requirements after suitable calibration methods. We intend to use the board in very diverse subjects in the next years; up to date, we have already included it in a control systems course (graduate) and in embedded realtime courses (undergraduate and master), as well as student dissertation projects and a web-accessible electronics-laboratory. Hence, we present the possibilities of our shield for these and other courses and also some educational results obtained in the past academic years.
\end{abstract}

Keywords: Microcontroller educational board, engineering training board, embedded real-time systems, control systems engineering.

\section{INTRODUCTION}

Laboratory work is a fundamental part of any engineering course. One of the most effective ways to guide students' training is by letting them experience the concepts they have seen in theory classes in the lab. This reinforces and enhances understanding of both theoretical and practical aspects of the subject, and provides a valuable glance into the kind of problems they might have to solve during their future professional careers. Hereof, there is a wide diversity of hardware devices on the market that are specifically aimed at laboratory practices, covering a wide range of subjects that go from general purpose (e.g. simple electric circuits) to more specific subjects (e.g. control and modulation of power inverters). In the case of electronic devices that can be used for training in engineering, we can group them roughly into four categories:

- Engineering training kits: These include all the devices that do not have a microcontroller or microprocessor exposed to the student (i.e. they are not openly programmable). They provide a number of pre-defined experiences for training in electronics [31],[8], control systems [17], and other fields of engineering. However, due to their lack of programming capabilities, they are not suitable for other important engineering disciplines, such as real-time systems, microcontroller programming, data acquisition, etc., that are limited to very specific exercises.

- Microcontroller training boards: They are printed circuit boards (PCB) that allow the user to plug-in a microcontroller unit (MCU) and provide a number of basic experiences for learning its 
use in embedded applications [27],[5],[25],[4],[30],[20]. To the best of the authors' knowledge, these solutions do not include experiences in control systems engineering, data acquisition or that involve external connections with different powered systems, as our proposal does; they are rather focused on digital interfaces and programming of the microcontroller, and are expensive.

- Microcontroller extension boards: These boards are cheaper than the previously described, since they extend an already existing microcontroller (possibly also integrated into a small board that provides power to the circuitry, as in the case of Arduino [22]). The training possibilities of these extension boards by themselves are therefore much more limited and specific than in the previous case [3],[9],[10], and definitely more limited than the solution proposed in this paper.

- Microprocessor training boards: They are analogous to their MCU counterpart, but use much more powerful processors (such as the popular Raspberry Pi [23]), thus they are more expensive than the former. Their main utility is for training on programming these CPUs, or for learning about operating systems, and therefore only provide digital interfaces with the external world, at most, thus limiting the kind of experiences that can be conducted in an engineering lab [13],[26].

Traditionally, most training and educational devices would fall into the first category, engineering training kits, as often encountered in many high-school and college labs, and as reflected by the great number of patents in the field [30],[20],[15],[28],[12],[11],[6]. These are rugged devices capable of withstanding rough use while also protecting the user from accidents, but they are also relatively expensive and proprietary, and thus out of reach for most learn-at-home students or schools in developing nations. In contrast, microcontroller training boards are much cheaper and accessible to the general public, and can be paired with extension boards to achieve higher functionalities. However, when it comes to training in engineering courses, the latter are still limited, as most are targeted at providing a single feature or solving a problem rather than on facilitating lab activities per se.

In this paper, we present a device that offers a very broad set of functionalities that can be used for engineering training: both $A D$ and $D A$ conversions, digital input/outputs, servomotor control and consumption measurement, external SRAM for data acquisition, SPI device for embedded communication practices, several built-in linear systems for control engineering, and connections to externally powered, +/-10v analog devices. Our device is a so-called shield for the popular openhardware Arduino board (i.e., it could be classified as a microcontroller extension board), therefore it takes advantage of the power source of Arduino, its embedded microcontroller (an 8-bit ATmega328P [24] @16MHz in the case of Arduino UNO [21]) and of its software ecosystem. Through this approach, we provide a solution that is significantly cheaper than dedicated hardware, as it extends Arduino, easily available at most laboratories and even in student's homes, whilst also providing the particular features we deemed most significant to facilitate education in engineering.

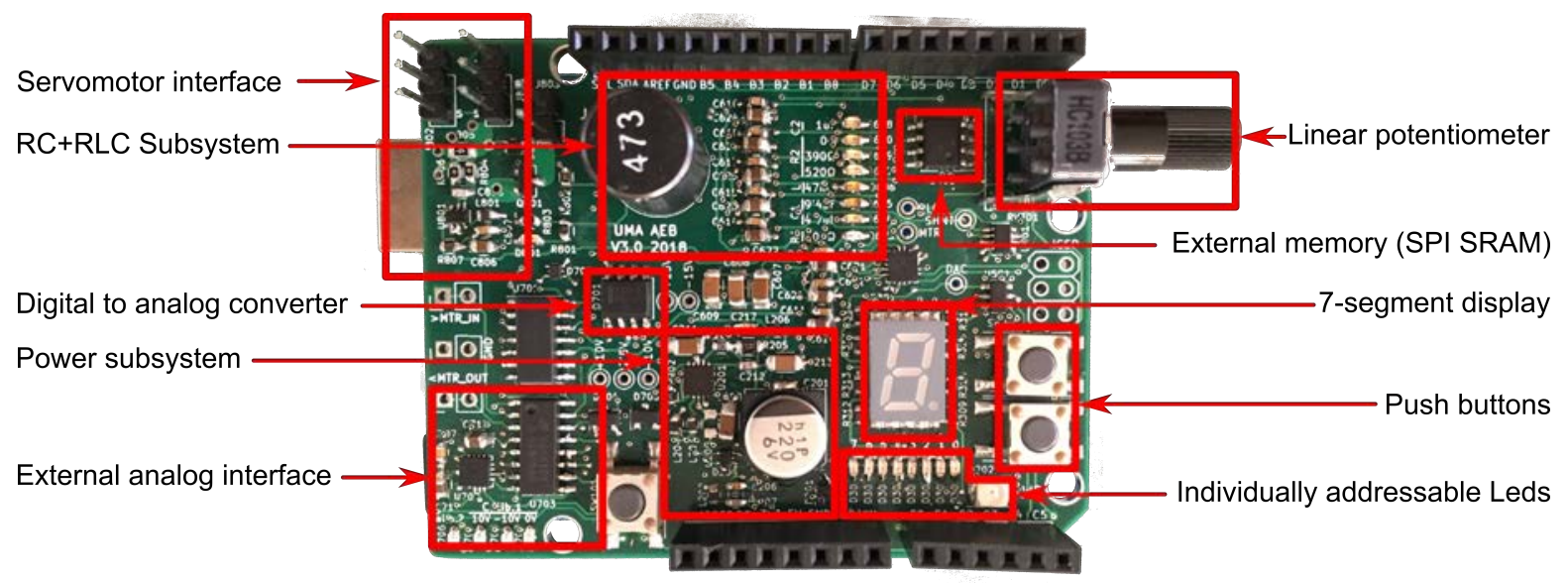

Figure 1. UMA Arduino Extension Board (UMA-AEB) and its subsystems.

Before this design, we have been using more primitive and limited versions since 2011; collecting numerous experiences and ideas on how to improve it to provide a better experience for the students as well as for the teachers that may incorporate it into their classes. Then, in the academic year 2016/2017, we designed the first manufactured version of our Arduino shield under an educational innovation project granted by the University of Málaga, and the year after we revised the design to incorporate the experiences gathered from its extensive use in courses from graduate and master levels: 
control engineering systems and embedded real-time systems, respectively. And soon after, we revised again some aspects of the board to improve its reliability and add new features [14]. The rest of this paper covers thus this last design (Fig. 1), with which we have been able to provide a diversity of exercises to the students that have fulfilled our initial requirements and expectations, and which we intend to use for a future study-from-home approach.

\section{OVERVIEW OF THE SHIELD}

The current version of the board and its main functionalities (see Fig. 1) are as follows:

- Power subsystem: Although our board gets power from the underlying Arduino (which, in turn, can drain it from a PC or an AC/DC wall power supply), we provide additional regulation and protections, in particular for the servomotor and other devices that can be directly powered from our shield.

- Led indicators and 7 segment display: This is a simple digital output subsystem composed of 9 low-consumption LEDs, which are physically arranged in a line to mimic the bits in a byte (plus an additional led for timing and debugging). It is mainly intended for training in bit manipulation programming, an essential topic in embedded systems, and for convenience when displaying information on the board.

- Buttons: We have included 2 independent buttons for digital user input. They are connected to interrupt signals in order to train for asynchronous real-time systems.

- Digital-Analog converter: This subsystem provides an analog output from a digital input composed of the same 8-bits connected to the light indicators. Note that this feature is not present on the Arduino board the shield connects to.

- External memory module: We have included in our design a $64 \mathrm{~KB}$ of SRAM that can be used for data acquisition (the RAM in the MCU is quite limited for that) and also for embedded communication practices, since it is connected to the MCU through a SPI bus.

- Linear potentiometer: A small rotational, linear potentiometer is included in the shield for simple analog readings (I.e. learning about analog-to-digital per se) or, once converted, as an input control signal to the program running on the Arduino.

- RC+RLC subsystem: This is maybe the most innovative and powerful subsystem in the shield: it consists of a resistive-inductive-capacitive (RLC) electrical circuit that, through a couple of switches, can be configured as a first-, second- or third order linear system with configurable constants. Moreover, it can be configured to run as an over-, critically-, or under-damped system in a continuous fashion so that the user may experiment with the different modes. Its electrical components have been chosen for allowing the MCU of the Arduino board to sample the output response appropriately. This subsystem is connected to the digital-analog converter, that produces its input, and to an analog input of Arduino, that receives the output, and can serve for data acquisition, system modeling, and system control exercises.

- External analog interface: This subsystem transforms the $[0,5] \vee$ range of the analog signals of the shield (e.g., those produced by the digital-analog converter) to and from a $[-10,+10] \mathrm{V}$ range, thus being useful for both acquiring and controlling external devices (that must be powered on their own), such as external DC motors that expect an analog signal to determine their speed and direction of rotation.

- External servomotor interface: This subsystem allows the user to connect typical RC servomotors or devices that are controlled with pulse-width modulated (PWM) signals generated by Arduino. In addition, a shunt resistor and an amplifier are inserted in the high-power rail in order to measure the consumption of such devices in real-time for subjects that cover forcecontrol on actuators.

All of the above features go through a double connector that, on one side, communicates with the Arduino, and, on the other side, exposes them to the users in case they want to also experiment with a breadboard. Moreover, to reduce the cost of manufacturing and to provide a footprint that is compatible with the original Arduino board, some subsystems share some of the control lines when their operation is not incompatible. Nonetheless, our shield can be used with other, newer, Arduino models. Aside from the footprint, the only considerations are that (i) our shield operates at $5 \mathrm{~V}$ and requires $500 \mathrm{~mA}$ minimum, although $1 \mathrm{~A}$ is recommended when using all components, (ii) the electric characteristics of 
the $R C+R L C$ resonator subsystem haven been chosen to be sampled by the ATmega328P running at $16 \mathrm{MHz}$, and (iii) the labeling of the connector on the shield correspond to MCU pin layout instead of the Arduino layout, which greatly facilitates programming without the Arduino IDE.

The cost for this design results in under $50 €$ when manufacturing a small batch of boards (around 10) by the students themselves, that is, $50 €$ for component acquisition and PCB manufacturing, but no soldering. We wager that manufacture and assembly of a higher volume might drop the cost to around $30 €$, leading to a retail price of under $100 €$.

\section{USING THE SHIELD IN ENGINEERING COURSES}

Table 1 lists the main engineering fields for which the shield can provide lab experiences, and how they can be set up by combining one or more of the subsystems described previously.

Table 1. Engineering fields for which the Shield is intended.

\begin{tabular}{l|l}
\hline \hline \multicolumn{1}{c|}{ Engineering Field } & \multicolumn{1}{c}{ Subsystem } \\
\hline \multirow{3}{*}{ Embedded systems } & Led indicators + display \\
& Buttons \\
& Linear potentiometer \\
& External servomotor interface \\
\hline Real-time embedded systems,, & Led indicators + display \\
Automation & Buttons \\
& External memory module \\
& Digital-Analog converter \\
& External servomotor interface \\
& External analog interface \\
\hline \multirow{5}{*}{ Data acquisition, Control } & Buttons \\
& External memory module \\
& Linear potentiometer \\
& Digital-Analog converter \\
& RC+RLC subsystem \\
& External analog interface \\
\hline \hline & Buttons \\
\hline \multirow{5}{*}{ Electrical engineering } & External memory module \\
& Linear potentiometer \\
& Digital-Analog converter \\
& RC+RLC subsystem \\
& External analog interface \\
& External servomotor interface \\
\hline & Digital-Analog converter \\
& External servomotor interface \\
& External analog interface \\
\hline & \\
\hline &
\end{tabular}

As commented in the introduction, we have employed intensively our board in several academic years, and in diverse engineering subjects. The list of subjects where the board is being or has been used, ECTS credits and other information is gathered in table II. 
Table 2. Engineering subjects using the Shield.

\begin{tabular}{l|c|l}
\hline \multicolumn{1}{c|}{ Subject } & Year / ECTS & \multicolumn{1}{c}{ Academic degree } \\
\hline Real Time for Mechatronic Systems & 1st / 29 & Master in Mechatronics Engineering \\
\hline Computer Control & 4 th / 18 & Degree in Computer Engineering \\
\hline Real Time Systems & 3rd / 24 & Degree in Computer Engineering \\
\hline Robot Programming & 3rd-4th / 21 & Various degrees of Computer Engineering \\
\hline Information Systems for Industry & 4th / 14 & Degree in Computer Engineering \\
\hline Automatic & 2nd / 73 & Degree in Industrial Technologies Engineering \\
\hline Automatic Control & 2nd / 68 & Degree in Health Engineering \\
\hline \hline
\end{tabular}

The exercises that use the shield vary considerably from subject to subject [7]. For instance, the syllabus of Real-Time Mechatronic Systems (master level) has a strong focus on embedded real-time systems [16], i.e., real-time programming for microcontrollers and hardware/software design and implementation of small-scale real-time systems. Accordingly, the shield was used in this subject for an exercise where the students had to command an RC servomotor to reach and hold a certain position with a PWM signal, and then sampling its energy consumption through the shield's shunt resistor, and compute and display the amount of force the motor is exerting on the 7-segment display. The gist of the exercise, however, was to implement all steps with an event-oriented code that must satisfy certain real-time requirements.

Another example, the Computer Control subject (degree level), which aims on acquiring data with the shield and displaying it in MATLAB to let the users experiment with LTI systems and their mathematical models. For instance, some of the exercises focused on various configuration of the RC+RLC circuitry, using it as a first, second and third order system that had to be tweaked (and mathematically verified) to fulfil certain criteria; whilst other of the exercises extended this idea to an external motor (controlled by the $\pm 10 \mathrm{~V}$ interface) to model its moment of inertia and damping coefficient.

\section{ACADEMIC RESULTS}

Regarding the evaluation of the impact that UMA-AEB has on our students, we have faced some significant limitations:

- The number of students enrolled in most of the courses is not large enough, especially when considering the enrollment fluctuations in some academic years.

- The low number of students, as well as some organizational limits, hampers the creation of a control group for most of the courses.

- The shield has been a work-in-progress development with several versions along the years. Besides, there has been some changes in the grading systems of some courses during this time. All of this affect to the comparisons of the results of different academic years.

Therefore, these constraints prevent a global and thorough statistical analysis of the influence of the UMA-AEB shield in the results obtained by our students. However, in order to have some measurement of the outcome of the shield in our teaching, we have conducted some partial statistical and qualitative reports that can be found in [7] and [2]; in the following we discuss some of the qualitative results shown in the latter.

We have run a survey among our students so they could assess their perception on some aspects before and after they used the shield in the lab sessions. From all the questions included in the survey, we focus here on those that reflect their evolution about Arduino and $C$ skills, the quality of the materials and attention provided by the teachers, how the shield affects their understanding of the theoretical concepts of the subject, and their experience with the shield. Fig. 2 (top) shows the results of the surveys for the students of the Real-Time in Mechatronics Systems, whereas Fig. 2 (bottom) shows the same information for the Computer Control subject.

In general, we see that students are satisfied with the shield. There is a positive trend in the histograms about changes in the Arduino and C skills in both subjects. Regarding the four other questions, the opinions of students of the Computer Control, while not bad, pointed out some problems. These problems were mostly due to the design of our practical exercises: mainly the lack of time due to the 
need of calibrating the board for each group; also, since the components were mounted on the board by ourselves in that first year, we detected a number of failures in some subsystems that consumed extra time for fixing and also produced some frustration on students when trying to do the exercises.

Considering all these aspects, we have obtained a very satisfactory experience when using the shield in all the courses: the students are more motivated, they are forced to practice, in a friendly and lowcost hardware/software environment, the concepts taught in theory classes, and, in general, obtain better results from the practical parts of the subject. The shield has proved also useful in that subjects that need to connect the Arduino to some specialized software, like ROS [29] (in the Robot Programming course) or LabVIEW [19] (in the Information Systems for Industry).

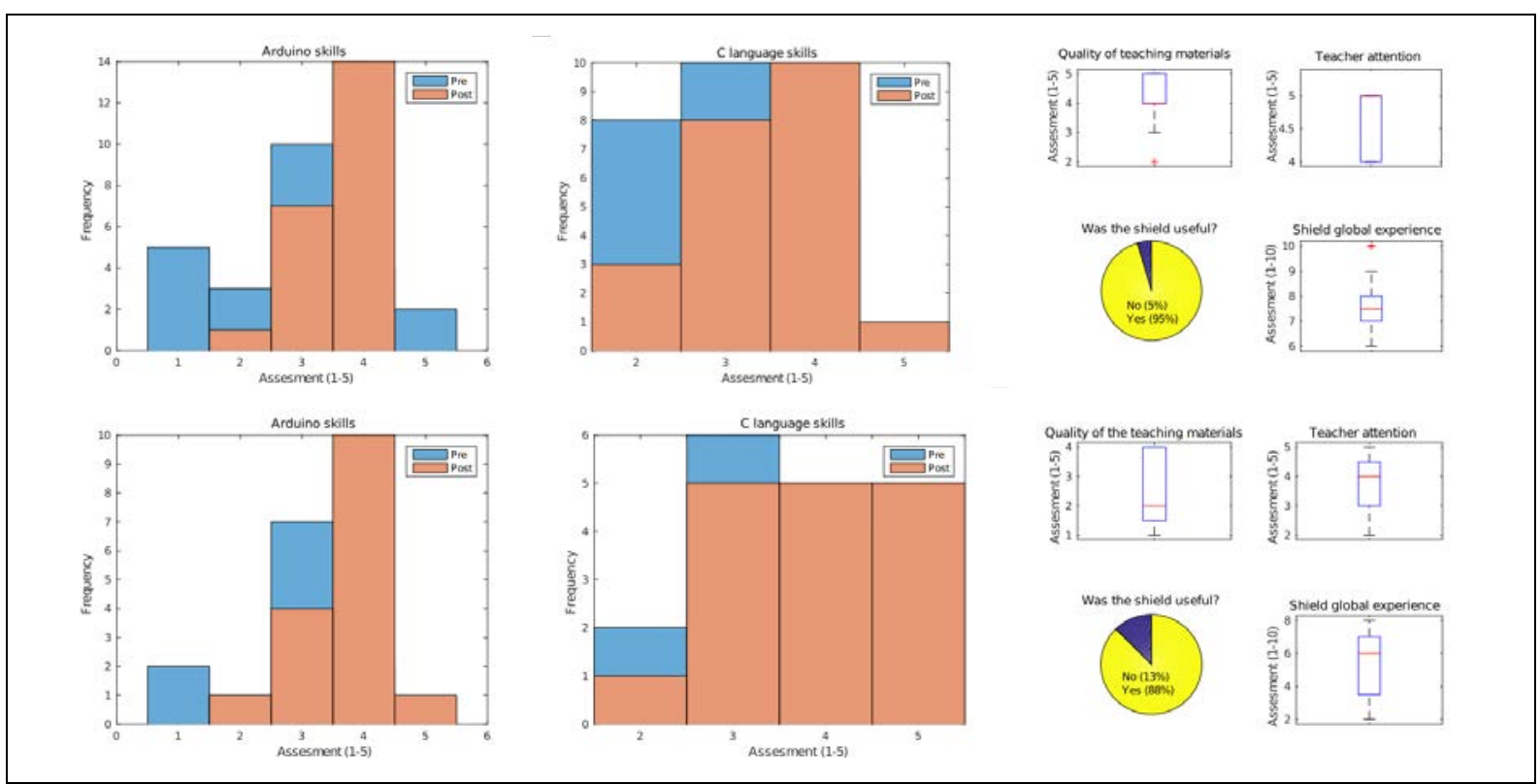

Fig. 2. Student questionary results from before and after introducing our shield to Real-Time Mechatronic Systems (master level), top, and Computer Control (degree level), bottom. From left to right: how the students auto evaluate their knowledge about Arduino and microcontrollers in general, how they evaluate their knowledge about the theory seen in the subjects, and various aspects of their academic performance.

\section{CONCLUSIONS AND FUTURE WORK}

In this paper we have presented a novel electronic shield for Arduino that, with a relatively low manufacture cost, provides in a single device a large number of training functionalities for engineering students. We have included a generic overview of the board and how we approach teaching engineering courses through it, that is, how it covers a wide range of fields: data acquisition, automatic control, realtime systems, robotics, etc.

For the future, we plan to reach a steady state in the use of the shields in the subjects that we are already using them, add new practical exercises and extend their use to different subjects. We also have plans on improving the design and adding new features to improve on its versatility. Moreover, we are already working on a full diagnosis software written in both $C$ and MATLAB that, through the connection of the board to a PC, will facilitate the use of the board through an additional abstraction layer so that subjects that are less coding oriented may use it more easily.

\section{ACKNOWLEDGEMENTS}

This work has been supported by the University of Málaga (Spain) through the educational innovation project PIE-15-093 "Innovación en el trabajo en laboratorio de una diversidad de asignaturas de ingeniería mediante el diseño y aplicación de una extensión de la plataforma de hardware abierto Arduino". 


\section{REFERENCES}

[1] A. Cruz-Martín, J.A. Fernández-Madrigal, C. Galindo, J. Gonzalez-Jimenez, C. Stockmans-Daou, J.L. Blanco, "A Lego Mindstorms NXT approach for teaching at data acquisition, control systems engineering and real-time systems undergraduate courses", Computers \& Education, vol. 59, no. 3, 2012.

[2] A. Gongora, J.A. Fernandez-Madrigal, A. Cruz-Martin, V. Arevalo, C. Galindo, C. Sanchez-Garrido, J. Monroy, J. Fernandez de Canete, "Shield Arduino de bajo coste para la enseñanza de asignaturas de Ingeniería", II Jornadas de Computación Empotrada y Reconfigurable (JCER2017), Málaga (Spain), pp. 1--10, 2017.

[3] Analog Shield. http://store.digilentinc.com/analog-shield-high-performance-add-on-board-for-thearduino-uno/ (16th Sept 2020).

[4] Basic STAMP Discovery Kit. https://www.parallax.com/product/27807. (16th Sept 2020).

[5] Board of Education Shield. https://www.parallax.com/product/35000. (16th Sept 2020).

[6] C. Durukan (2014). Training and experiment system supported by an animation based full simulation method. Code WO2015112103A1.

[7] C. Galindo, J.A. Fernandez-Madrigal, "Grounding Concepts and Methods of Real-Time Scheduling in Reality using Arduino", IEEE Transactions on Education, vol. 63, no. 3, pp. 224-231, 2020.

[8] Circuits for training in electronics. https://shop.elenco.com/consumers/brands/snap-circuits.html. Consulted 16th sept. 2020.

[9] DAC Shield. https://www.tindie.com/products/visgence/power-dac-shield/ (16th Sept 2020).

[10] Digital and Analog 10 Expander Shield. http://numato.com/digital-and-analog-io-expander-shield/ (16th Sept 2020).

[11] E.Z. Gabriel (1974). Electronic analog computers. Code US3996457A.

[12] E.Z. Gabriel (1979). Educational analog computer laboratory. Code US4315320A.

[13] Gertboard for Raspberry Pi. http://es.farnell.com/gertboard/gertboard/assembled-gertboard-forraspberry/dp/2250034 (16th Sept 2020).

[14] J. Martínez-Lahoz, J.A. Fernandez-Madrigal, and A. Gongora. "Desarrollo Integral y Ensayos del escudo Docente UMA_AEB_V2.00". Master Dissertation at the University of Malaga, 2019.

[15] K. Jensen (1982). Simulator systems for interactive simulation of complex dynamic systems. Code US4464120A.

[16] Laplante P.A. (2004), Real-Time Systems Design and Analysis, IEEE Press, ISBN 0-471-22855-9.

[17] Lucas-Nuelle company page. http://www.lucas-nuelle.com. (16th Sept 2020).

[18] N.S. Nise (2011). Control Systems Engineering. International Student Version, Sixth Edition, Wiley, ISBN 978-0-470-64612-0.

[19] NI LabVIEW (2020). http://www.ni.com. (21th Sept 2020).

[20] NIDA Corp. Electronic teaching and testing device. Code US4213253A.

[21] Official website of Arduino/Genuino UNO (2016). Official website of the Arduino/Genuino UNO, https://www.arduino.cc/en/Main/ArduinoBoardUno. (16th Sept 2020)

[22] Official website of Arduino/Genuino, https://www.arduino.cc. (16th Sept 2020).

[23] Official website of Raspberry Pi. https://www.raspberrypi.org. (16th Sept 2020).

[24] Official website of the ATmega328P microcontroller, http://www.atmel.com/devices/atmega328p.aspx. (16th Sept 2020).

[25] PIC Programmer \& Experiment Kit K8048. https://www.velleman.eu/products/view/?id=350903 (16th Sept 2020).

[26] Piface I/O board for Raspberry Pi. http://es.farnell.com/piface/piface-digital/i-o-expansion-board-forraspberry/dp/2218566 (16th Sept 2020). 
[27] Portable Dual Arduino (TM) Micro XPlorerBoard. https://www.kickstarter.com/projects/ 1576747460/portable-dual-arduino-micro-xplorerboard (16th Sept 2020).

[28] R. M. Kuczewski (1996). Cooperative/interactive learning system for logic instruction. Code US5868575A.

[29] Robot Operating System (2020). http://www.ros.org. (16th Sept 2020).

[30] Sony Corp. Interactive teaching apparatus. Code US4812125A.

[31] SparkFun inventor kit. https://www.sparkfun.com/products/12060. (16th Sept 2020). 